\title{
Enhanced DET-Based Fault Signature Analysis for Reliable Diagnosis of Single and Multiple-Combined Bearing Defects
}

\author{
In-Kyu Jeong, ${ }^{1}$ Myeongsu Kang, ${ }^{1}$ Jaeyoung Kim, ${ }^{1}$ Jong-Myon Kim, ${ }^{1}$ \\ Jeong-Min $\mathrm{Ha}^{2}$ and Byeong-Keun $\mathrm{Choi}^{2}$ \\ ${ }^{1}$ School of Electrical, Electronics and Computer Engineering, University of Ulsan, Ulsan 680-749, Republic of Korea \\ ${ }^{2}$ Department of Energy and Mechanical Engineering, Gyeongsang National University, Tongyeong 650-160, Republic of Korea
}

Correspondence should be addressed to Jong-Myon Kim; jongmyon.kim@gmail.com

Received 30 January 2015; Revised 16 May 2015; Accepted 25 May 2015

Academic Editor: Wei Xu

Copyright (C) 2015 In-Kyu Jeong et al. This is an open access article distributed under the Creative Commons Attribution License, which permits unrestricted use, distribution, and reproduction in any medium, provided the original work is properly cited.

To early identify cylindrical roller bearing failures, this paper proposes a comprehensive bearing fault diagnosis method, which consists of spectral kurtosis analysis for finding the most informative subband signal well representing abnormal symptoms about the bearing failures, fault signature calculation using this subband signal, enhanced distance evaluation technique- (EDET-) based fault signature analysis that outputs the most discriminative fault features for accurate diagnosis, and identification of various single and multiple-combined cylindrical roller bearing defects using the simplified fuzzy adaptive resonance map (SFAM). The proposed comprehensive bearing fault diagnosis methodology is effective for accurate bearing fault diagnosis, yielding an average classification accuracy of $90.35 \%$. In this paper, the proposed EDET specifically addresses shortcomings in the conventional distance evaluation technique (DET) by accurately estimating the sensitivity of each fault signature for each class. To verify the efficacy of the EDET-based fault signature analysis for accurate diagnosis, a diagnostic performance comparison is carried between the proposed EDET and the conventional DET in terms of average classification accuracy. In fact, the proposed EDET achieves up to $106.85 \%$ performance improvement over the conventional DET in average classification accuracy.

\section{Introduction}

Although rolling element bearings are the most significant elements to support heavy loads in rotating machines [1], they frequently faced severe failures during operation. Because these unscheduled bearing defects cause tremendous economic losses, reliable bearing fault diagnosis is urgently demanded. Thus, we propose a comprehensive bearing fault diagnosis method that includes the following two essential aspects: fault signature extraction and pattern recognition.

As a failure occurs in a rolling element bearing, it is hard to extract fault characteristic information from the nonstationary vibration signals [2]. To address this nonstationary issue, time-frequency analyses have become an indispensable part of fault diagnosis of bearings. These include shorttime Fourier transform (STFT) [3, 4], wavelet transform (WT) [5-9], and empirical mode decomposition (EMD) [10-17]. Traditionally, STFT has been used in processing nonstationary signals by carrying out a fast Fourier transform (FFT) for short periods of time; this determines which frequency is present at which time. However, this gives a constant resolution for all the frequencies because it requires a constant length window for the entire signal, which produces a uniform partition of the time-frequency plane. In general, abnormal symptoms of the rolling element bearing will appear anywhere in the mid-/high-frequency spectrum range. This requires multiresolution analysis, which gives a fine time resolution and a coarse frequency resolution at high frequencies, while giving a fine frequency resolution but a coarse time resolution for low frequencies. Hence, STFT has been superseded by both WT and EMD. Although both WT and EMD are effective time-frequency decomposition tools, which are widely applied to reliable bearing fault diagnosis, they have a common shortcoming. When a signal is decomposed into multiple subband signals and intrinsic mode functions (IMFs) by WT and EMD, respectively, it is 


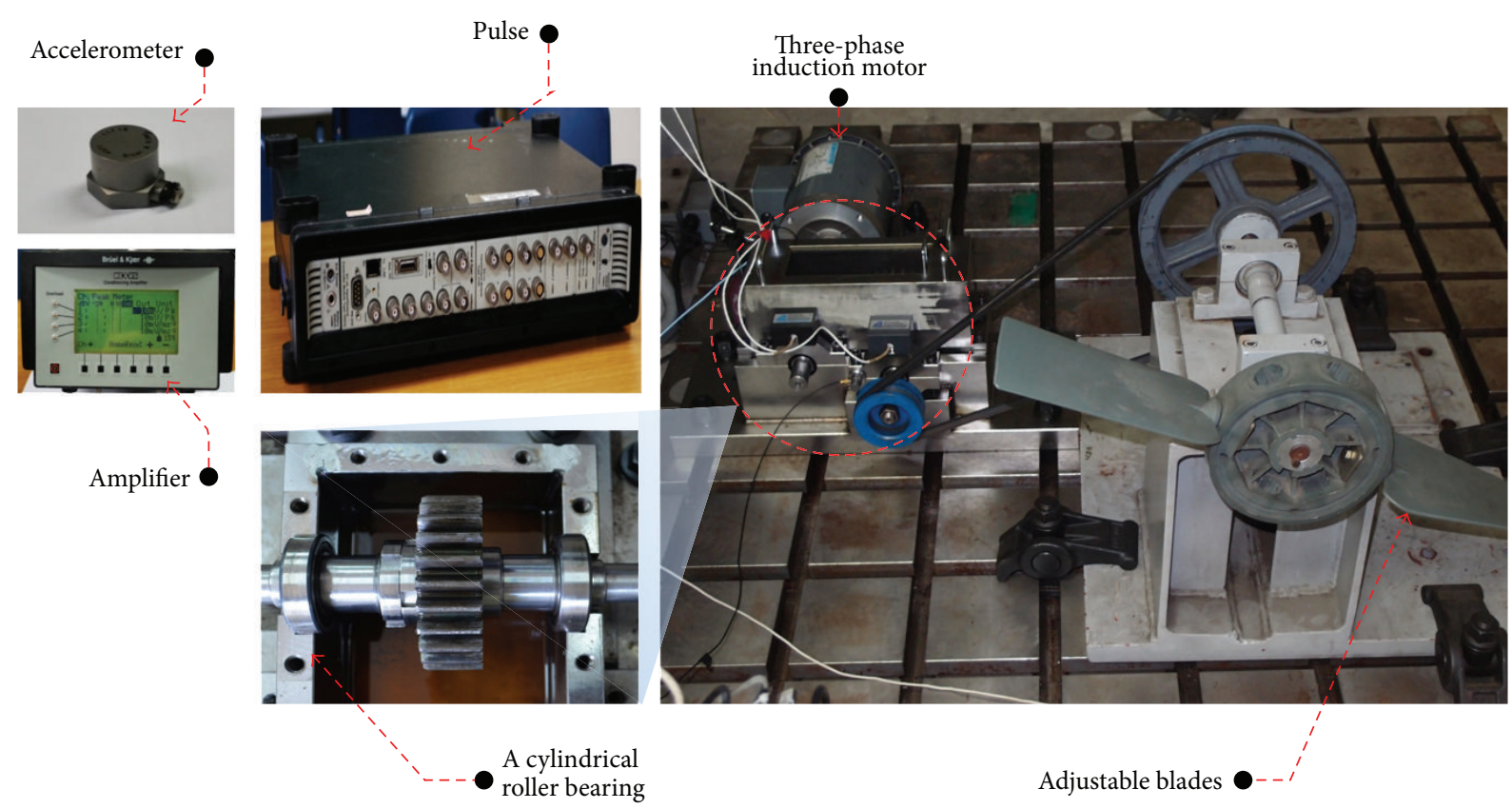

FIgURE 1: A self-designed fault simulator.

necessary to select informative subband signals and IMFs that contain intrinsic information about various bearing defects. To deal with this issue, this paper employs spectral kurtosis analysis (i.e., kurtogram) [18] in our comprehensive bearing fault diagnosis approach and it is performed by calculating kurtosis values from envelope power spectra, where envelope power spectra are obtained from the decomposed subband signals at different wavelet packet decomposition depths. Based on these spectral kurtosis measurements, we exploit a subband signal yielding the highest spectral kurtosis value for feature extraction.

Recently, the importance of fault feature analysis has gradually increased because all the extracted fault signatures may not be equally useful for accurate diagnosis. Furthermore, a high-dimensional feature vector can be a primary reason of the diagnostic performance degradation. To deal with high-dimensional, non-Gaussian fault signatures, Jin et al. used trace ratio linear discriminant analysis and showed satisfactory results to diagnose various bearing failures [19]. Jiang et al. proposed semisupervised kernel marginal fisher analysis for optimal feature selection by reducing incompetent features [20]. They fed the optimal low-dimensional features into a $k$-nearest neighbor classifier to recognize different fault categories of bearing defects. Principal component analysis- (PCA-) based bearing fault prediction methods are also familiar [21]. Derivatives of PCA in a nonlinear form, such as kernel PCA (KPCA) and kernel fisher discriminant analysis (KFDA), have been used in fault diagnosis to allow nonlinear dependency between feature variables [22-25]. Shen et al. employed a distance evaluation technique (DET) for sensitive fault-feature selection [26], which usually exhibits a large degree of variance for samples belonging to different classes and a smaller degree of variance for samples belonging to the same class. For reliable bearing fault diagnosis, this paper proposes a derivative of DET, enhanced DET (EDET), to decide the most discriminative subset of all the extract fault signatures. The conventional DET has drawbacks in sensitivity estimation of each fault feature because it is based on average intraclass compactness and interclass separability among different classes. In practice, the average is not effective for dealing with skewed features. Hence, the EDET modifies the sensitivity estimation scheme in the conventional DET for improving feature analysis efficiency. To identify various bearing failures, we use a simplified fuzzy ARTMAP (SFAM), a special class of artificial neural networks, as the classifier [27-29], where ARTMAP denotes an adaptive resonance theory map.

The rest of the paper is organized as follows. Section 2 describes a self-designed fault simulator including the data acquisition system and seeded bearing failures considered in this study. Section 3 introduces the comprehensive bearing fault diagnosis scheme, and Section 4 shows experimental results. Finally, Section 5 concludes this paper.

\section{A Self-Designed Fault Simulator and Seeded Bearing Failures}

Figure 1 shows a self-designed fault simulator involving a data acquisition system, an induction motor, a gearbox whose reduction ration is $1.52: 1$, and adjustable blades. Likewise, either a defect-free cylindrical roller bearing (DFCRB) or defective cylindrical roller bearings can be installed in the nondrive end bearing housing of this self-designed fault simulator. To capture abnormal symptoms of seeded bearings, this paper attaches a piezoelectric accelerometer (type 4371) to the nondrive end bearing housing and continuously records vibration signals sampled at $65.536 \mathrm{kHz}$ by using 
TABLE 1: Summary of specifications of the data acquisition system.

\begin{tabular}{ll}
\hline $\begin{array}{l}\text { NEXUS conditioning amplifier } \\
\text { (type 2692-C) }\end{array}$ & $\begin{array}{l}\text { (i) Frequency range: } \\
0.1 \mathrm{~Hz}-100 \mathrm{kHz} \\
\text { (ii) Sensitivity: } 10 \mathrm{mV} / \mathrm{ms}^{-2}\end{array}$ \\
\hline Piezoelectric charge & (i) Frequency range: \\
accelerometer (type 4371) & $0.1 \mathrm{~Hz}-12.6 \mathrm{kHz}$ \\
& (ii) Sensitivity: $9.8 \mathrm{pC} / \mathrm{g}$ \\
\hline $\begin{array}{l}\text { Portable data acquisition device } \\
\text { (PULSE type 3560-C) }\end{array}$ & $\begin{array}{l}\text { Maximum sampling } \\
\text { frequency: } 25.6 \mathrm{kHz}\end{array}$ \\
\hline
\end{tabular}

an amplifier (NEXUS condition amplifier type 2692-C) and a portable data acquisition device (PULSE type 3560-C) because vibration analysis is effective for analyzing dynamic activities in bearing failures [30-34]. Table 1 summaries specifications of the data acquisition system. Although vibration signals can provide intrinsic information about bearing failures, unwanted random noise, which may be nonlinear and non-Gaussian, inherent in the measured vibration signals can result in diagnostic performance deterioration. To address this issue, we employ a Gaussian particle filter that is effective for solving the non-Gaussian noise problem. More details about its measurement and time update procedures for estimating non-Gaussian noise are provided in [35].

To verify the proposed comprehensive bearing fault diagnosis methodology, this paper obtains 120 vibration signals for each bearing (i.e., a DFCRB and seven different defective bearings) that rotates at the shaft speed of 482 revolutionsper-minute (RPM) under slight load condition, as presented in Table 2. In addition, various single and multiple-combined bearing defects are depicted in Figure 2.

\section{Proposed Bearing Fault Diagnosis Methodology}

The proposed comprehensive bearing fault diagnosis approach is composed of the following two processes: analysis process and evaluation process. The analysis process of the bearing fault diagnosis scheme is to decide an optimal subset of all the extracted statistical fault signatures while the evaluation process is to validate efficacy of the proposed diagnosis approach to identify incipient bearing defects. Likewise, the analysis dataset includes randomly selected 30 vibration signals for each bearing condition and the remaining vibration signals are used for the evaluation dataset in this study. Figure 3 pictorially illustrates the overall process of the proposed bearing fault diagnosis methodology.

3.1. Analysis Process. An optimal subset of fault signatures is determined via this analysis process, which consists of spectral kurtosis analysis, feature pool configuration, and discriminative feature selection using the enhanced distance evaluation technique (EDET).

Step 1. Practically, abnormal symptoms of incipient bearing defects are well revealed in mid- or high-frequency subband signals due to the amplitude modulation [36]. This calls the exploration of subband signals that involve intrinsic information about the bearing defects. To address this issue, we exploit spectral kurtosis analysis [18], which outputs the most informative subband signal yielding the highest kurtosis value of the kurtogram that is generated by measuring kurtosis of envelop power spectra calculated from subband signals. In this paper, the subband signals are obtained by means of discrete wavelet packet transform with a Daubechies 45-tap filter (e.g., db45). Furthermore, an envelope power spectrum is yielded by squaring the magnitude of fast Fourier transform of an envelope signal, where the envelope signal is the absolute value of the complex-valued analytical signal that is obtained by combining the given time-domain subband signal and its Hilbert transformed signal. Figure 4 depicts an example of spectral kurtosis analysis for a CRBCO.

Although a spectral kurtosis measurement is effective for quantifying the amplitudes of the bearing characteristic frequency (or bearing defect frequency) and its harmonics in the envelope power spectrum, this measurement, which basically measures the degree of protrusion, can be influenced by unwanted spectral peaks such as harmonics of operating frequency and spectral peaks that are not relevant to bearing defects. Hence, we compute a kurtosis value for the envelope power spectrum ranging from $N \times F_{o}$ to $N \times$ $\max (\mathrm{BPFO}, \mathrm{BPFI}, 2 \times \mathrm{BSF})$ to produce the kurtogram as shown in Figure 4, where $N$ ( $N=3$ in this study) is an arbitrary natural number, $F_{o}$ is the shaft speed in Hertz that is computed by RPM/60, BPFO is the ball pass frequency for the outer raceway, BPFI is the ball pass frequency for the inner raceway, and BSF is the ball spin frequency, respectively, and the bearing defect frequencies (e.g., BPFO, BPFI, and BSF) are defined as follows [34]:

$$
\begin{aligned}
\mathrm{BPFO} & =\frac{N_{r} \cdot F_{o}}{2}\left(1-\frac{B_{d}}{P_{d}} \cos \alpha\right), \\
\mathrm{BPFI} & =\frac{N_{r} \cdot F_{o}}{2}\left(1+\frac{B_{d}}{P_{d}} \cos \alpha\right), \\
\mathrm{BSF} & =\frac{P_{d} \cdot F_{o}}{2 \cdot B_{d}}\left(1-\left(\frac{B_{d}}{P_{d}} \cos \alpha\right)^{2}\right) .
\end{aligned}
$$

These bearing characteristic frequencies are computed with the following parameters: the number of cylindrical rollers $\left(N_{r}=13\right)$, the shaft speed in $\operatorname{Hertz}\left(F_{o}=8.03 \mathrm{~Hz}\right)$, the contact angle $\left(\alpha=0^{\circ}\right)$, and roller and pitch diameters $\left(B_{d}=9 \mathrm{~mm}\right.$ and $\left.P_{d}=46.5 \mathrm{~mm}\right)$. Namely, BPFO $=42.09 \mathrm{~Hz}, \mathrm{BPFI}=$ $62.30 \mathrm{~Hz}$, and $2 \times \mathrm{BSF}=39.93 \mathrm{~Hz}$, respectively.

Step 2. A $N_{\text {classes }} \times N_{\text {samples }} \times N_{\text {features }}$ feature pool, $F$, is configured in Step 2 by calculating statistical parameters that are widely used for bearing fault diagnosis, where $N_{\text {classes }}$ is the number of classes to be discriminated in this study (i.e., a DFCRB and seven defective bearings), $N_{\text {samples }}$ is the number of data samples for each bearing condition in the analysis dataset $\left(N_{\text {samples }}=30\right.$ in this study), and $N_{\text {features }}$ is the total number of fault signatures that are defined in Table 3. A moment is mathematically defined as a specific quantitative measure for the shape of a set of points. Thus, statistical moments such as mean (first moment), standard deviation (second moment about the mean), skewness (third moment), 
TABLE 2: Seeded bearing failures considered in this study.

\begin{tabular}{ll}
\hline CRBCO & A cylindrical roller bearing with a crack on its outer raceway \\
CRBCI & A cylindrical roller bearing with a crack on its inner raceway \\
CRBCR & A cylindrical roller bearing with a crack on its roller \\
CRBCOI & A cylindrical roller bearing with a crack on its outer and inner raceways \\
CRBCOR & A cylindrical roller bearing with a crack on its outer raceway and roller \\
CRBCIR & A cylindrical roller bearing with a crack on its inner raceway and roller \\
CRBCOIR & A cylindrical roller bearing with a crack on its outer raceway, inner raceway, and roller \\
Crack size & \\
Length & $12 \mathrm{~mm}$ on a bearing's outer and inner raceways and 10 mm on a bearing's roller \\
Width & $0.49 \mathrm{~mm}$ \\
$\quad$ Depth & $0.5 \mathrm{~mm}$ \\
Average RPM & 482 \\
\hline
\end{tabular}

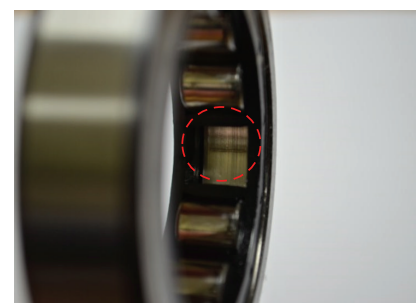

(a)

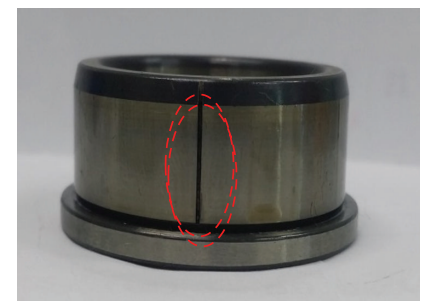

(b)

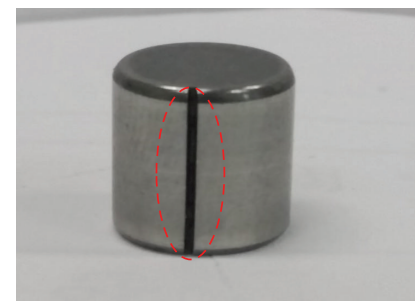

(c)

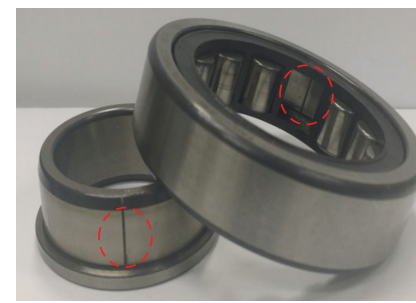

(d)

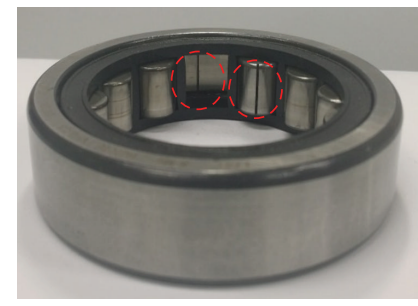

(e)

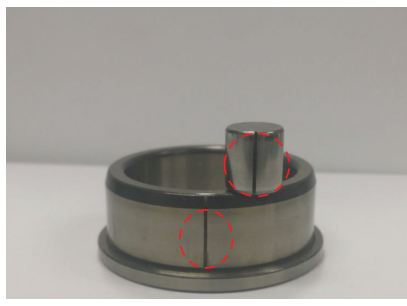

(f)

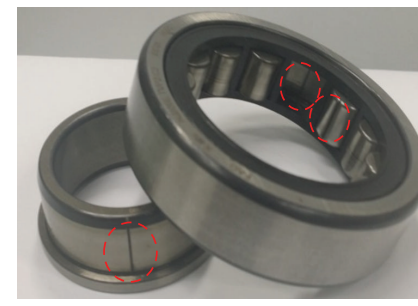

(g)

Figure 2: Single and multiple-combined bearing defects. (a) CRBCO, (b) CRBCI, (c) CRBCR, (d) CRBCOI, (e) CRBCOR, (f) CRBCIR, and (g) CRBCOIR.

Analysis process

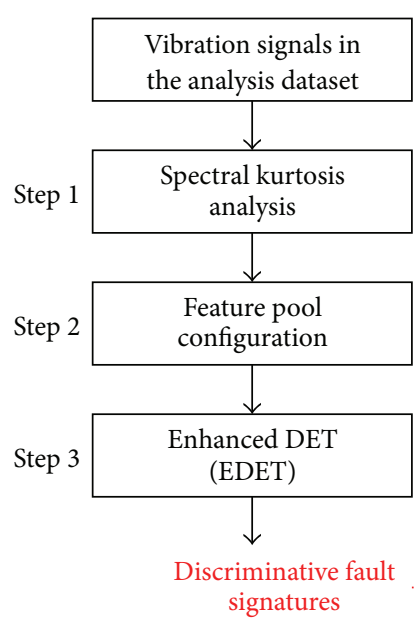

Evaluation process

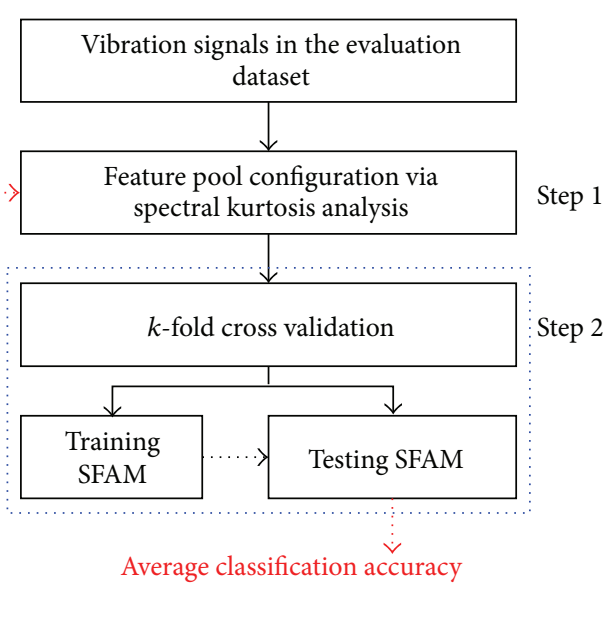

FIGURE 3: Overall process of the proposed comprehensive bearing fault diagnosis methodology. 
TABLE 3: Twelve statistical fault signatures, where $x$ is the most informative subband signal via spectral kurtosis analysis, $N$ is the total number of the subband signal, $x_{\text {rms }}$ is the root mean square value of $x$, and $\max (|x|)$ outputs the maximum value of $x$.

\begin{tabular}{lccc}
\hline Parameter & Definition & Parameter & Definition \\
\hline Mean $\left(f_{1}\right)$ & $\bar{x}=\frac{1}{N} \sum_{i=1}^{N} x_{i}$ & Crest factor $\left(f_{2}\right)$ & max $(x)$ \\
\hline Impulse factor $\left(f_{3}\right)$ & $\frac{\max (|x|)}{(1 / N) \sum_{i=1}^{N}\left|x_{i}\right|}$ & Standard deviation $\left(f_{4}\right)$ & $\frac{1}{N} \sum_{i=1}^{N}\left(x_{i}-\bar{x}\right)^{2}$ \\
\hline Kurtosis $\left(f_{5}\right)$ & $\frac{(1 / N) \sum_{i=1}^{N}\left(x_{i}-\bar{x}\right)^{4}}{\left((1 / N) \sum_{i=1}^{N}\left(x_{i}-\bar{x}\right)^{2}\right)^{2}}$ & Skewness $\left(f_{6}\right)$ & $(1 / N) \sum_{i=1}^{N}\left(x_{i}-\bar{x}\right)^{3}$ \\
\hline Energy $\left(f_{7}\right)$ & $\frac{(1 / N) \sum_{i=1}^{N}\left(x_{i}-\bar{x}\right)^{5}}{\left((1 / N) \sum_{i=1}^{N}\left(x_{i}-\bar{x}\right)^{2}\right)^{5 / 2}}$ & Peak $\left(f_{8}\right)$ & max $(|x|)$ \\
\hline 5th normalized moment $\left(f_{9}\right)$ & $\frac{\max (|x|)}{\left((1 / N) \sum_{i=1}^{N} \sqrt{\left|x_{i}\right|}\right)^{2}}$ & Absolute mean amplitude value $\left(f_{12}\right)$ & $\frac{1}{N} \sum_{i=1}^{N} x_{i}^{2}$ \\
Latitude factor $\left(f_{11}\right)$ & $\frac{1}{N} \sum_{i=1}^{N}\left|x_{i}\right|$
\end{tabular}

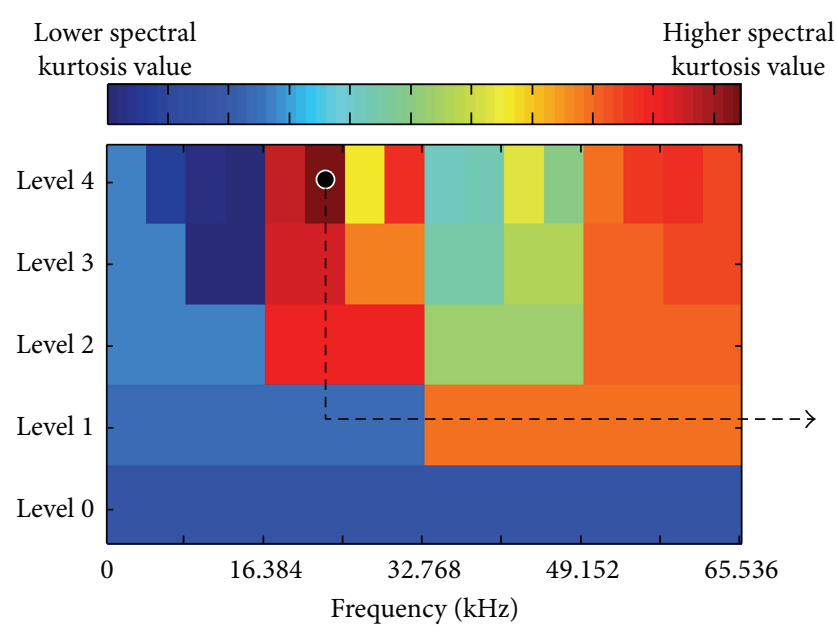

FIGURE 4: An example of spectral kurtosis analysis for a CRBCO. kurtosis (forth moment), and 5th moment can provide useful information for discriminative single and multiple-combined bearing defects. Moreover, peak, energy, and absolute mean amplitude values are used as key fault signatures for bearing fault diagnosis due to their effectiveness quantifying the severe bearing failures. In particular, peak and crest factor, defined as the ratio of the peak level of the vibration signal to the root mean square (RMS) level, are widely used to detect changes of the impulsive vibrations due to BCO. The RMS, defined as a quantitative measure of the power content in the vibration signal, is also effective for detecting incipient bearing defects.

Step 3. Although the main goal of feature selection is to reduce computation burden caused by a large number of fault signatures, feature selection can be often useful for

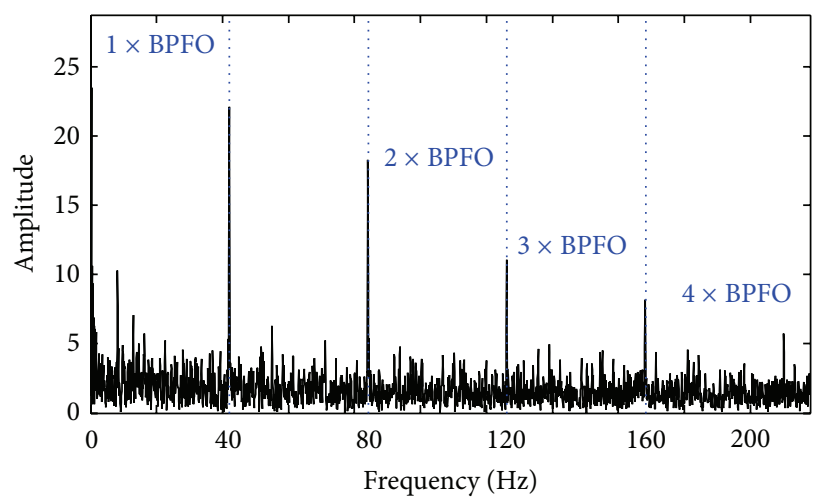

Harmonics of BPFO accurate bearing fault diagnosis by removing some of them that do not contribute much to identification of bearing failures. Thus, this paper proposes the EDET, an improvement on DET [26], to determine an optimal subset of fault signatures. As discussed in Section 1, the conventional DET estimates degree of sensitivity of each fault signature to decide whether it is a discriminative feature minimizing intraclass compactness and maximizing the interclass separability. To meausre the sensitivity of the $i$ th fault signature, the DET first calculates the intraclass compactness for the $i$ th fault signature by compactness $i=\left(1 / N_{\text {classes }}\right) \sum_{m=1}^{N_{\text {classes }}} D_{m, i}$, where $D_{m, i}$ indicates the degree of the $i$ th feature density for the $m$ th class which is expressed as $D_{m, i}=\left(1 /\left(N_{\text {samples }}\right.\right.$. $\left.\left.\left(N_{\text {samples }}-1\right)\right)\right) \sum_{j=1}^{N_{\text {samples }}} \sum_{k=1, j \neq k}^{N_{\text {samples }}}|F(m, j, i)-F(m, k, i)|$ and then computes the interclass separability for the $i$ th fault 

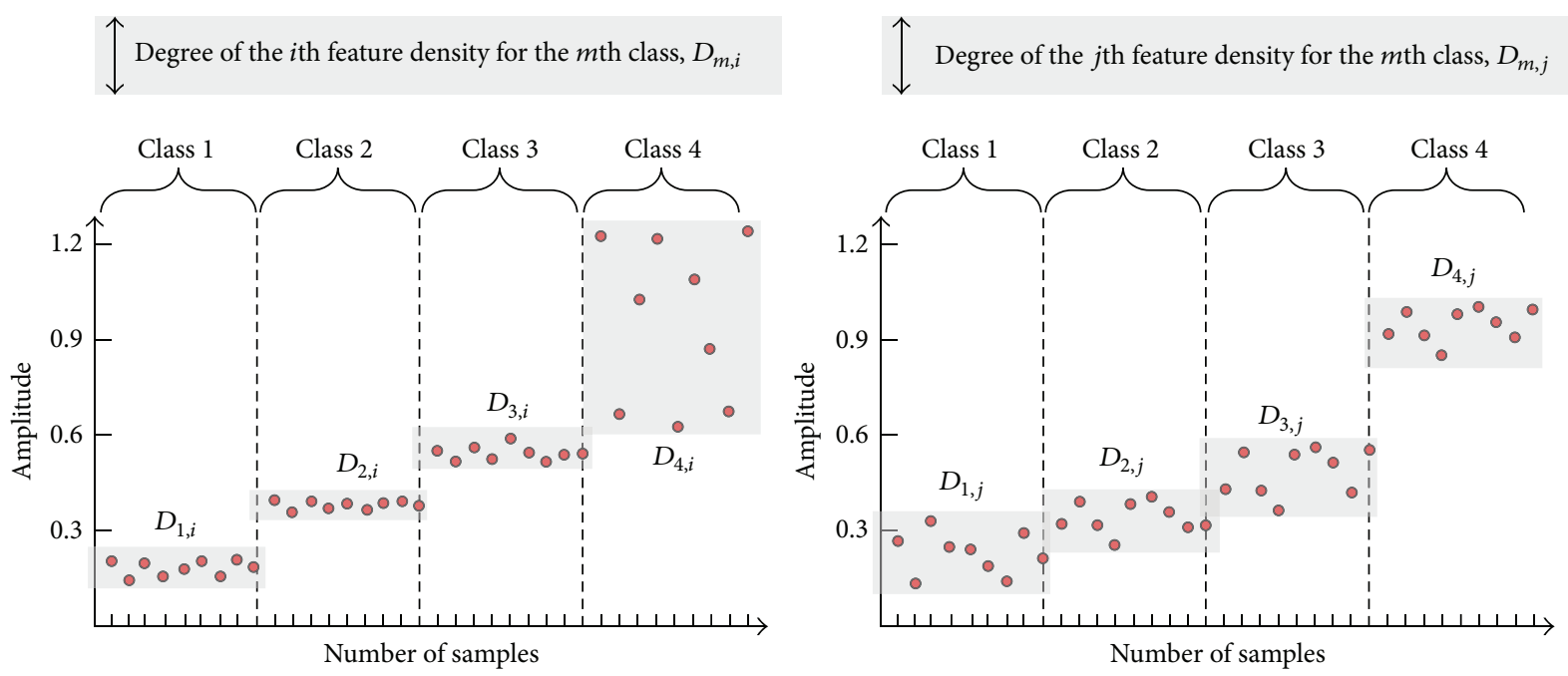

FigurE 5: Examples of the $i$ th and $j$ th fault signature distribution per class with the assumption that these features have the same degrees of the interclass separability.

signature by seperability ${ }_{i}=\left(1 /\left(N_{\text {classes }} \cdot\left(N_{\text {classes }}-\right.\right.\right.$ 1))) $\sum_{m=1}^{N_{\text {classes }}} \sum_{n=1, m \neq n}^{N_{\text {classes }}}\left|C_{m, i}-C_{n, i}\right|$, where $C_{m, i}$ is the centroid value of the $i$ th fault signature for the $m$ th class that is defined as $C_{m, i}=\left(1 / N_{\text {samples }}\right) \sum_{k=1}^{N_{\text {samples }}} F(m, k, i)$. Finally, the sensitivity of the $i$ th fault signature, $\alpha_{i}$, is expressed as $\alpha_{i}=$ seperability $_{i} /$ compactness $_{i}$. In general, the conventional DET finding a subset of fault signatures that yield higher sensitivities shows satisfactory performance for bearing fault diagnosis. However, the DET has the following shortcomings when estimating the sensitivity of each fault signature:

(i) In the conventional DET, an average value of the degrees of feature density for all classes is used to measure the intraclass compactness and this intraclass compactness measurement can be drawback that the sensitivity of fault signatures is misestimated. Figure 5 illustrates examples of the $i$ th and $j$ th fault signature distribution per class with the assumption that these two feature have the same degrees of the interclass separability. As shown in Figure 5, although the ith fault feature is more useful for discriminating the first three classes (i.e., class 1, class 2, and class 3) rather than the $j$ th fault signature, a very low degree of the $i$ th feature density for the fourth class (i.e., class 4) may greatly decrease the sensitivity of this feature, where a low degree of the $i$ th feature density for the $m$ th class (or a higher value of $D_{m, i}$ ) implies the $i$ th feature is widely dispersed.

(ii) Similar to the intraclass compactness estimation in the conventional DET, the interclass separability using an average distance among centroids of each class is another shortcoming in sensitivity estimation of fault signatures. Figure 6 depicts examples of the $i$ th and $j$ th fault feature distribution with the assumption that these signatures have the same degrees of the intraclass compactness. In Figure 6, the sensitivity of the $j$ th fault feature can be much higher than the $i$ th feature due to the great distance between a centroid of the fourth class and centroids of the other classes. According to the feature selection policy of the conventional DET, the $j$ th fault signature is selected for fault diagnosis. Figure 6, however, shows the $i$ th fault feature is more helpful for identifying these classes than the $j$ th signature.

Accordingly, this paper enhances the conventional DET (EDET) by measuring the sensitivity of each fault signature for each class. To do this, we use the $i$ th feature density for the $m$ th class, $D_{m, i}$, as the intraclass compactness of the $i$ th fault signature for the $m$ th class, compactness $s_{m, i}$, and estimate the interclass separability of the $i$ th feature for the $m$ th class, separability ${ }_{m, i}$, by computing separability ${ }_{m, i}=$ $\min _{n \neq m}\left(\left|C_{m, i}-C_{n, i}\right|\right), m, n=1,2, \ldots, N_{\text {classes }}$, where $\min ()$ is the minimum value of its argument. Finally, the sensitivity of the $i$ th feature for the $m$ th class, $\alpha_{m, i}$, is defined as $\alpha_{m, i}=$ separability $_{m, i} /$ compactness $_{m, i}$. In this study, the minimum dimension of the discriminative feature subset can be one if fault features yielding the highest sensitivity for each class are the same, whereas the maximum dimension of the discriminative feature subset can be $N_{\text {classes }}$ if fault features selected by the proposed EDET are different from each other.

3.2. Evaluation Process. As shown in Figure 3, the evaluation process verifies the effectiveness of the proposed bearing fault diagnosis methodology, which consists of the following two steps.

Step 1. This paper configures a feature pool by carrying out spectral kurtosis analysis for vibration signals in the evaluation dataset (i.e., 90 vibration signals for each bearing condition), selecting informative subband signals, and calculating statistical parameters determined by the EDET in the analysis process from these subband signals.

Step 2. As mentioned in Section 1, the SFAM is used to identify various single and multiple-combined low-speed bearing failures in this study. Likewise, $k$-fold cross validation [37] is 

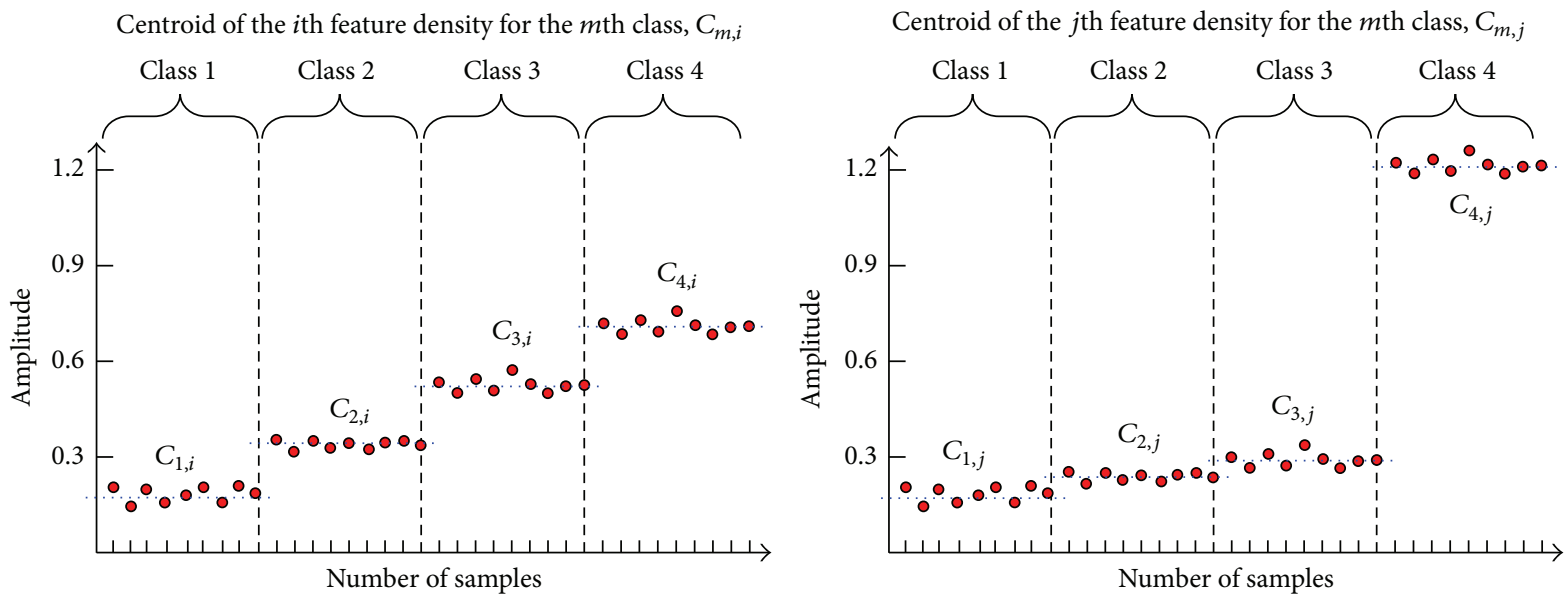

FiguRE 6: Examples of the $i$ th and $j$ th fault signature distribution per class with the assumption that these features have the same degrees of the intraclass compactness.

employed to estimate the generalized diagnostic performance (or classification accuracy) of the proposed comprehensive bearing fault diagnosis approach. In $k$-fold cross validation, both training and testing the classifier are repeated $k$ times by using randomly partitioned $k$ mutual folds from the feature pool in the evaluation process, denoted by $F_{1}, F_{2}, \ldots, F_{k}$. Namely, the classifier (i.e., SFAM) is trained in the fold $F_{i}$ and tested in the remaining folds at the $i$ th iteration of $k$-fold cross validation. Hence, the diagnostic performance of the bearing fault diagnosis approach is estimated by computing average values of classification accuracies and true positive rates resulting from each iteration in $k$-fold cross validation, where $k$ is set to 3 for accurate diagnostic performance estimation in this study. Both average classification accuracy (ACA) and average true positive rate (ATPR) are defined as follows:

$$
\begin{aligned}
\mathrm{ACA} & =\frac{1}{k} \sum_{i=1}^{k} \mathrm{CA}_{i}, \\
\mathrm{ATPR} & =\frac{1}{k} \sum_{i=1}^{k} \mathrm{TPR}_{i},
\end{aligned}
$$

where both $\mathrm{CA}_{i}$ and $\mathrm{TPR}_{i}$ are the classification accuracy and true positive rate resulting at the $i$ th iteration of $k$-fold cross validation, which are defined as CA = $\left(\sum_{N_{\text {classes }}} N_{\mathrm{TP}} / N_{\text {datasamples }}\right) \times 100(\%)$ and $\mathrm{TPR}=\left(N_{\mathrm{TP}} /\left(N_{\mathrm{TP}}+\right.\right.$ $\left.\left.N_{\mathrm{FN}}\right)\right)^{2} \times 100(\%)$, respectively. Likewise, $N_{\mathrm{TP}}$ and $N_{\mathrm{FN}}$ are the number of true positives (e.g., a true positive is when a sample in the "CRBCO" class is correctly classified as the "CRBCO" class) and false negatives (e.g., a false negative is when a sample not in the "CRBCO" class is not classified as the "CRBCO" class).

\section{Experimental Results}

In this paper, the EDET-based feature signature analysis is used for effectively identifying single and multiple-combined bearing defects and this section validates its efficacy in terms of ACA and ATPR. As mentioned in Section 3.1, because the conventional DET determines a subset of fault features

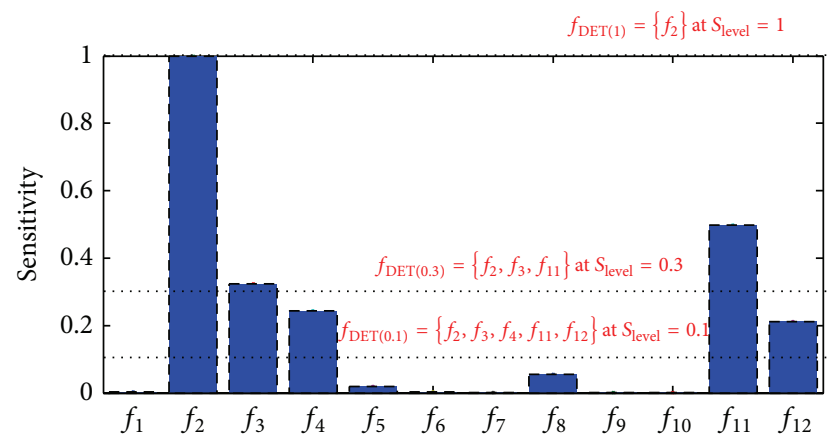

FIGURE 7: Sensitivity of fault features by means of the conventional DET.

based on the sensitivity level $\left(S_{\text {level }}\right)$, it is necessary to explore the impacts of various subsets of fault signatures on the diagnostic performance, where fault signatures with high sensitivity are useful for diagnosis in the DET-based feature selection scheme. Table 4 shows a performance comparison between the conventional DET and the proposed EDET and the sensitivity level of all the extracted fault signatures in this study is illustrated in Figure 7. In Table 5, the conventional DET shows an average classification accuracy of $43.68 \%$, $84.96 \%$, and $84.58 \%$ for $S_{\text {level }}=1, S_{\text {level }}=0.3$, and $S_{\text {level }}=0.1$, respectively, where the subsets of fault features for $S_{\text {level }}=1$, $S_{\text {level }}=0.3$, and $S_{\text {level }}=0.1$ are $f_{\text {DET(1) }}=\left\{f_{2}\right\}$, $f_{\mathrm{DET}(0.3)}=\left\{f_{2}, f_{3}, f_{11}\right\}$, and $f_{\mathrm{DET}(0.1)}=\left\{f_{2}, f_{3}, f_{4}, f_{11}, f_{12}\right\}$. An interesting observation in Table 4 is that the average classification accuracy is even worse $(43.68 \%)$ at $S_{\text {level }}=$ 1. Accordingly, the conventional DET is inefficient for estimating the sensitivity of each fault feature. In contrast, the proposed EDET approach achieves an average classification accuracy of $90.35 \%$, where the most discriminative subset of fault signatures $f_{\mathrm{EDET}}=\left\{f_{1}, f_{5}, f_{7}, f_{12}\right\}$.

Although the EDET is effective for improving the diagnostic performance by sorting out discriminative fault signatures of twelve fault features, the bearing defects are not sufficiently classified between CRBCO and CRBCOR, as presented in Table 5. Figure 8 depicts a 3-dimensional visualization result of the three fault features (i.e., $f_{5}, f_{7}$, and 


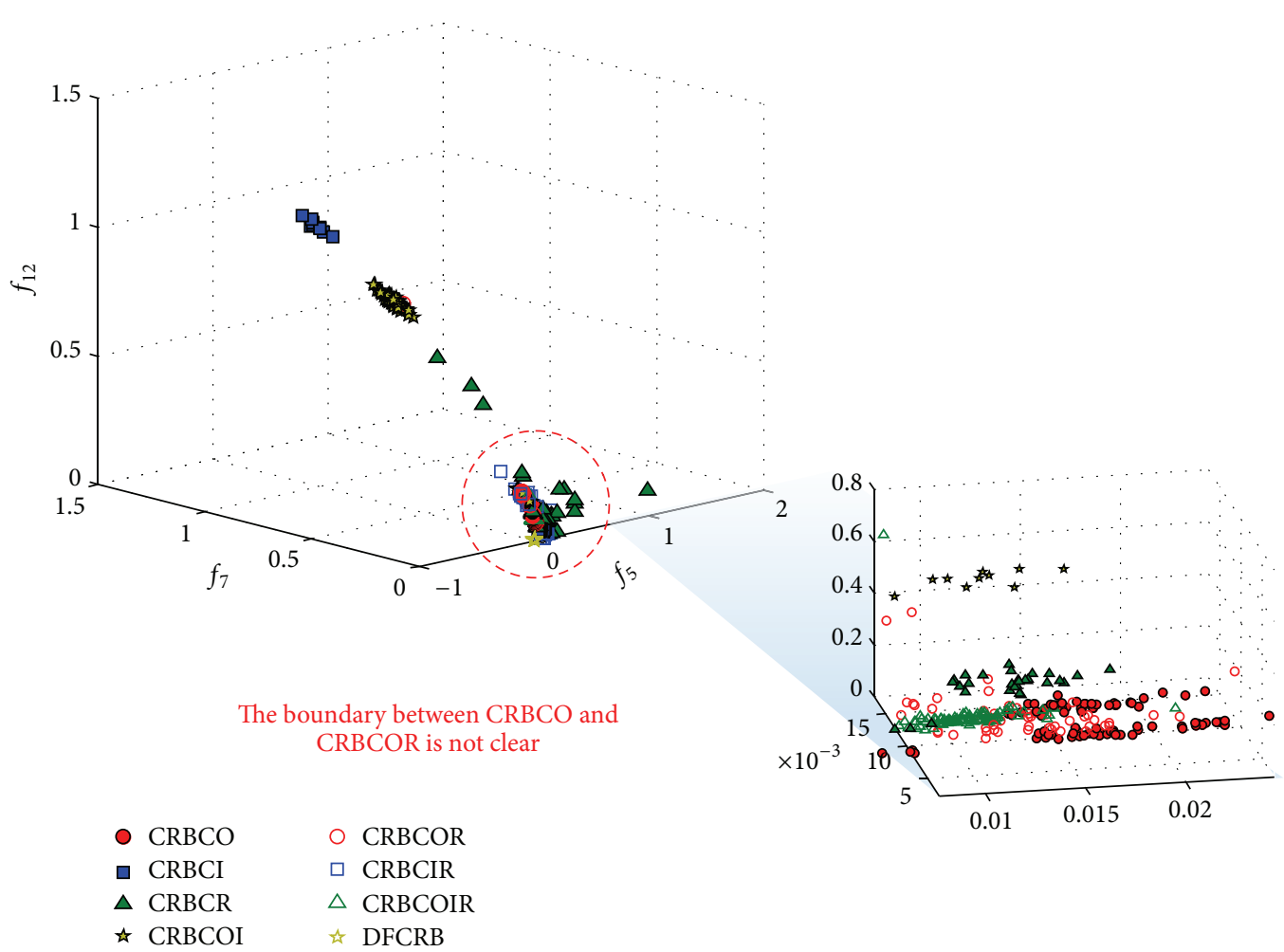

FIGURE 8: 3-dimensional visualization result of the three fault features in $f_{\mathrm{EDET}}=\left\{f_{1}, f_{5}, f_{7}, f_{12}\right\}$.

TABLE 4: Performance comparison between the conventional DET and the proposed EDET in terms of average classification accuracy and true positive rate via $k$-fold cross validation (Unit: \%).

\begin{tabular}{|c|c|c|c|c|c|c|c|c|c|c|}
\hline & \multirow{2}{*}{$S_{\text {level }}$} & \multicolumn{8}{|c|}{ ATPR for each bearing condition (standard deviation) } & \multirow{2}{*}{$\begin{array}{c}\text { ACA } \\
\text { (standard deviation) }\end{array}$} \\
\hline & & $\mathrm{CRBCO}$ & CRBCI & CRBCR & CRBCOI & CRBCOR & CRBCIR & $\mathrm{CR}$ & $\mathrm{RB}$ & \\
\hline \multirow{3}{*}{ DET } & 1 & $27.22(6.74)$ & $37.22(9.18)$ & $43.33(4.41)$ & $51.11(10.72)$ & $22.22(5.09)$ & $35.00(2.89)$ & $33.33(5.77)$ & $100.00(0.00)$ & $43.68(5.60)$ \\
\hline & 0.3 & $82.22(5.36)$ & $76.67(2.89)$ & $93.33(2.89)$ & $93.89(2.55)$ & $74.44(9.18)$ & $75.00(10.41)$ & $83.33(11.67)$ & & 62) \\
\hline & 0.1 & $85.00(7.26)$ & $73.33(1.67)$ & $93.33(4$. & $93.89(2.55)$ & $67.22(5$ & & & 100. & 3.63) \\
\hline EDET & - & $91.67(2.89)$ & $84.44(3.50)$ & $96.11(5.36)$ & $93.33(2.89)$ & 76.67 (11.67) & $86.67(7.26)$ & $93.89(0.96)$ & $100.00(0.00)$ & $90.35(4.31)$ \\
\hline
\end{tabular}

TABLE 5: Classification result of the proposed bearing fault diagnosis method using a confusion matrix, where $k$-fold cross validation is performed.

\begin{tabular}{|c|c|c|c|c|c|c|c|c|}
\hline & \multicolumn{8}{|c|}{ Test outcome } \\
\hline & $\mathrm{CRBCO}$ & CRBCI & CRBCR & CRBCOI & CRBCOR & CRBCIR & CRBCOIR & DFCRB \\
\hline \multicolumn{9}{|l|}{ Condition } \\
\hline CRBCO & 165 & 0 & 2 & 0 & 8 & 3 & 2 & 0 \\
\hline CRBCI & 0 & 173 & 2 & 0 & 0 & 5 & 0 & 0 \\
\hline CRBCR & 0 & 1 & 152 & 2 & 5 & 16 & 4 & 0 \\
\hline CRBCOI & 0 & 0 & 1 & 168 & 0 & 11 & 0 & 0 \\
\hline CRBCOR & 29 & 0 & 7 & 2 & 138 & 0 & 4 & 0 \\
\hline CRBCIR & 1 & 8 & 7 & 8 & 0 & 156 & 0 & 0 \\
\hline CRBCOIR & 4 & 0 & 2 & 1 & 4 & 0 & 169 & 0 \\
\hline DFCRB & 0 & 0 & 0 & 0 & 0 & 0 & 0 & 100 \\
\hline
\end{tabular}


$\left.f_{12}\right)$ in $f_{\mathrm{EDET}}=\left\{f_{1}, f_{5}, f_{7}, f_{12}\right\}$. As shown in Figure 8 , the boundary between these two bearing defects heavily overlaps and is unclear, resulting in degraded diagnostic performance. Thus, it is needed to extract a new fault feature preserving intrinsic information about a CRBCOR to increase its true positive rate.

\section{Conclusion}

In this study, a comprehensive bearing fault diagnosis approach is presented to diagnose single and multiplecombined cylindrical roller bearing defects. This approach first finds the most informative subband signal via the discrete wavelet packet transform-based spectral kurtosis analysis and configures a feature pool by calculating twelve statistical parameters in the subband signal. However, all the extracted fault signatures may be equally useful for accurately identifying various bearing failures. Accordingly, the EDETbased fault signature analysis is carried out to determine an optimal subset of fault features, which is further used for training and testing the SFAM. Experimental results indicate that the proposed fault diagnosis method is effective for accurate bearing fault diagnosis. In fact, the EDET achieves up to $106.85 \%$ performance improvement over the conventional DET in ACA.

\begin{tabular}{|c|c|}
\hline \multicolumn{2}{|c|}{ bbreviation } \\
\hline ACA: & Average classification accuracy \\
\hline ATPR: & Average true positive rate \\
\hline BPFI: & $\begin{array}{l}\text { A ball pass frequency for the inner } \\
\text { raceway }\end{array}$ \\
\hline BPFO: & $\begin{array}{l}\text { A ball pass frequency for the outer } \\
\text { raceway }\end{array}$ \\
\hline BSF: & A ball spin frequency \\
\hline CRBCI: & $\begin{array}{l}\text { A cylindrical roller bearing with a crack } \\
\text { on its inner raceway }\end{array}$ \\
\hline CRBCIR: & $\begin{array}{l}\text { A cylindrical roller bearing with a crack } \\
\text { on its inner raceway and roller }\end{array}$ \\
\hline CRBCO: & $\begin{array}{l}\text { A cylindrical roller bearing with a crack } \\
\text { on its outer raceway }\end{array}$ \\
\hline CRBCOI: & $\begin{array}{l}\text { A cylindrical roller bearing with a crack } \\
\text { on its outer and inner raceways }\end{array}$ \\
\hline CRB & $\begin{array}{l}\text { A cylindrical roller bearing with a crack or } \\
\text { its outer raceway, inner raceway, and rolle }\end{array}$ \\
\hline CRBCOR: & $\begin{array}{l}\text { A cylindrical roller bearing with a crack } \\
\text { on its outer raceway and roller }\end{array}$ \\
\hline CRBCR: & $\begin{array}{l}\text { A cylindrical roller bearing with a crack } \\
\text { on its roller }\end{array}$ \\
\hline DFCRB: & A defect-free cylindrical roller bearing \\
\hline DET: & Distance evaluation technique \\
\hline EDET: & Enhanced distance evaluation technique \\
\hline EMD: & Empirical mode decomposition \\
\hline FFT: & Fast Fourier transform \\
\hline IMF: & Intrinsic mode function \\
\hline KFDA: & Kernel fisher discriminant analysis \\
\hline KPCA: & Kernel principal component analysis \\
\hline PCA: & Principal component analysis \\
\hline RPM: & Revolutions-per-minute \\
\hline
\end{tabular}

SFAM: Simplified fuzzy adaptive resonance theory map

STFT: Short-time Fourier transform

WT: Wavelet transform.

\section{Conflict of Interests}

The authors declare that there is no conflict of interests regarding the publication of this paper.

\section{Acknowledgments}

This work was supported by a National Research Foundation of Korea (NRF) grant funded by the Korea government (MSIP) (no. NRF-2013R1A2A2A05004566) and by the Korean government (MEST) (no. 2012R1A1A2043644). This research was also financially supported by Korea Aerospace Industries Ltd. (KAI), the Ministry of Trade, Industry \& Energy (MOTIV), Korea Institute for Advancement of Technology (KIAT)n and Dongnam Institute for Regional Program Evaluation through the Leading Industry Development for Economic Region.

\section{References}

[1] M. Kang, J. Kim, A. C. Tan, E. Y. Kim, and B. Choi, "Reliable fault diagnosis for low-speed bearings using individually trained support vector machines with kernel discriminative feature analysis," IEEE Transactions on Power Electronics, vol. 30, no. 5, pp. 2786-2797, 2015.

[2] H. L. Ao, J. Cheng, K. Li, and T. K. Truong, "A roller bearing fault diagnosis method based on LCD energy entropy and ACROASVM," Shock and Vibration, vol. 2014, Article ID 825825, 12 pages, 2014.

[3] E. Cabal-Yepez, A. G. Garcia-Ramirez, R. J. Romero-Troncoso, A. Garcia-Perez, and R. A. Osornio-Rios, "Reconfigurable monitoring system for time-frequency analysis on industrial equipment through STFT and DWT,' IEEE Transactions on Industrial Informatics, vol. 9, no. 2, pp. 760-771, 2013.

[4] M. Cocconcelli, R. Zimroz, R. Rubini, and W. Bartelmus, "STFT based approach for ball bearing fault detection in a varying speed motor," in Condition Monitoring of Machinery in NonStationary Operations, pp. 41-50, Springer, Berlin, Germany, 2012.

[5] D. Bianchi, E. Mayrhofer, M. Gröschl, G. Betz, and A. Vernes, "Wavelet packet transform for detection of single events in acoustic emission signals," Mechanical Systems and Signal Processing, 2015.

[6] Y. Wang, G. Xu, L. Liang, and K. Jiang, "Detection of weak transient signals based on wavelet packet transform and manifold learning for rolling element bearing fault diagnosis," Mechanical Systems and Signal Processing, vol. 54-55, pp. 259-276, 2015.

[7] P. K. Kankar, S. C. Sharma, and S. P. Harsha, "Fault diagnosis of rolling element bearing using cyclic autocorrelation and wavelet transform," Neurocomputing, vol. 110, pp. 9-17, 2013.

[8] P. K. Kankar, S. C. Sharma, and S. P. Harsha, "Rolling element bearing fault diagnosis using wavelet transform," Neurocomputing, vol. 74, no. 10, pp. 1638-1645, 2011. 
[9] R. Yan, R. X. Gao, and X. Chen, "Wavelets for fault diagnosis of rotary machines: a review with applications," Signal Processing, vol. 96, pp. 1-15, 2014.

[10] A. Djebala, M. K. Babouri, and N. Ouelaa, "Rolling bearing fault detection using a hybrid method based on empirical mode decomposition and optimized wavelet multi-resolution analysis," The International Journal of Advanced Manufacturing Technology, 2015.

[11] X. Liu, L. Bo, and H. Luo, "Bearing faults diagnostics based on hybrid LS-SVM and EMD method," Measurement, vol. 59, pp. 145-166, 2015.

[12] M. Zhao, J. Lin, X. Xu, and X. Li, "Multi-fault detection of rolling element bearings under harsh working condition using IMFbased adaptive envelope order analysis," Sensors, vol. 14, no. 11, pp. 20320-20346, 2014.

[13] Y. Yuan, X. Zhao, J. Fei, Y. Zhao, and J. Wang, "Study on fault diagnosis of rolling bearing based on time-frequency generalized dimension," Shock and Vibration. In press.

[14] L. Saidi, J. B. Ali, and F. Fnaiech, "Bi-spectrum based-EMD applied to the non-stationary vibration signals for bearing faults diagnosis," ISA Transactions, vol. 53, no. 5, pp. 1650-1660, 2014.

[15] W. Guo and P. W. Tse, "A novel signal compression method based on optimal ensemble empirical mode decomposition for bearing vibration signals," Journal of Sound and Vibration, vol. 332, no. 2, pp. 423-441, 2013.

[16] C. Junsheng, Y. Dejie, and Y. Yu, "A fault diagnosis approach for roller bearings based on EMD method and AR model," Mechanical Systems and Signal Processing, vol. 20, no. 2, pp. 350362, 2006.

[17] D. Yu, J. Cheng, and Y. Yang, "Application of EMD method and Hilbert spectrum to the fault diagnosis of roller bearings," Mechanical Systems and Signal Processing, vol. 19, no. 2, pp. 259270, 2005.

[18] D. Wang, P. W. Tse, and K. L. Tsui, "An enhanced Kurtogram method for fault diagnosis of rolling element bearings," Mechanical Systems and Signal Processing, vol. 35, no. 1-2, pp. 176-199, 2013.

[19] X. Jin, M. Zhao, T. W. S. Chow, and M. Pecht, "Motor bearing fault diagnosis using trace ratio linear discriminant analysis," IEEE Transactions on Industrial Electronics, vol. 61, no. 5, pp. 2441-2451, 2014.

[20] L. Jiang, J. Xuan, and T. Shi, "Feature extraction based on semisupervised kernel Marginal Fisher analysis and its application in bearing fault diagnosis," Mechanical Systems and Signal Processing, vol. 41, no. 1-2, pp. 113-126, 2013.

[21] S. Dong and T. Luo, "Bearing degradation process prediction based on the PCA and optimized LS-SVM model," Measurement, vol. 46, no. 9, pp. 3143-3152, 2013.

[22] K. Feng, Z. Jiang, W. He, and B. Ma, "A recognition and novelty detection approach based on Curvelet transform, nonlinear PCA and SVM with application to indicator diagram diagnosis," Expert Systems with Applications, vol. 38, no. 10, pp. 12721-12729, 2011.

[23] M. Žvokelj, S. Zupan, and I. Prebil, "Non-linear multivariate and multiscale monitoring and signal denoising strategy using kernel principal component analysis combined with ensemble empirical mode decomposition method," Mechanical Systems and Signal Processing, vol. 25, no. 7, pp. 2631-2653, 2011.

[24] V. H. Nguyen and J.-C. Golinval, "Fault detection based on Kernel principal component analysis," Engineering Structures, vol. 32, no. 11, pp. 3683-3691, 2010.
[25] Z.-B. Zhu and Z.-H. Song, "A novel fault diagnosis system using pattern classification on kernel FDA subspace," Expert Systems with Applications, vol. 38, no. 6, pp. 6895-6905, 2011.

[26] C. Shen, D. Wang, F. Kong, and P. W. Tse, "Fault diagnosis of rotating machinery based on the statistical parameters of wavelet packet paving and a generic support vector regressive classifier," Measurement: Journal of the International Measurement Confederation, vol. 46, no. 4, pp. 1551-1564, 2013.

[27] J. B. Ali, L. Saidi, A. Mouelhi, B. Chebel-Morello, and F. Fnaiech, "Linear feature selection and classification using PNN and SFAM neural networks for a nearly online diagnosis of bearing naturally progressing degradations," Engineering Applications of Artificial Intelligence, vol. 42, pp. 67-81, 2015.

[28] M. Jin, R. Li, Z. Xu, and X. Zhao, "Reliable fault diagnosis method using ensemble fuzzy ARTMAP based on improved Bayesian belief method," Neurocomputing, vol. 133, pp. 309-316, 2014.

[29] G. A. Carpenter, S. Grossberg, N. Markuzon, J. H. Reynolds, and D. B. Rosen, "Fuzzy ARTMAP: a neural network architecture for incremental supervised learning of analog multidimensional maps," IEEE Transactions on Neural Networks, vol. 3, no. 5, pp. 698-713, 1992.

[30] J. Seshadrinath, B. Singh, and B. K. Panigrahi, "Investigation of vibration signatures for multiple fault diagnosis in variable frequency drives using complex wavelets," IEEE Transactions on Power Electronics, vol. 29, no. 2, pp. 936-945, 2014.

[31] M. Cococcioni, B. Lazzerini, and S. L. Volpi, "Robust diagnosis of rolling element bearings based on classification techniques," IEEE Transactions on Industrial Informatics, vol. 9, no. 4, pp. 2256-2263, 2013.

[32] M. D. Prieto, G. Cirrincione, A. G. Espinosa, J. A. Ortega, and $\mathrm{H}$. Henao, "Bearing fault detection by a novel conditionmonitoring scheme based on statistical-time features and neural networks," IEEE Transactions on Industrial Electronics, vol. 60, no. 8, pp. 3398-3407, 2013.

[33] I. Bediaga, X. Mendizabal, A. Arnaiz, and J. Munoa, "Ball bearing damage detection using traditional signal processing algorithms," IEEE Instrumentation and Measurement Magazine, vol. 16, no. 2, pp. 20-25, 2013.

[34] I. Bediaga, X. Mendizabal, I. Etxaniz, and J. Munoa, "An integrated system for machine tool spindle head ball bearing fault detection and diagnosis," IEEE Instrumentation and Measurement Magazine, vol. 16, no. 2, pp. 42-47, 2013.

[35] J. H. Kotecha and P. M. Djuric, "Gaussian particle filtering," IEEE Transactions on Signal Processing, vol. 51, no. 10, pp. 25922601, 2003.

[36] G. Dong, J. Chen, and F. Zhao, "A frequency-shifted bispectrum for rolling element bearing diagnosis," Journal of Sound and Vibration, vol. 339, pp. 396-418, 2015.

[37] J. D. Rodriguez, A. Perez, and J. A. Lozano, "Sensitivity analysis of k-fold cross validation in prediction error estimation," IEEE Transactions on Pattern Analysis and Machine Intelligence, vol. 32, no. 3, pp. 569-575, 2010. 

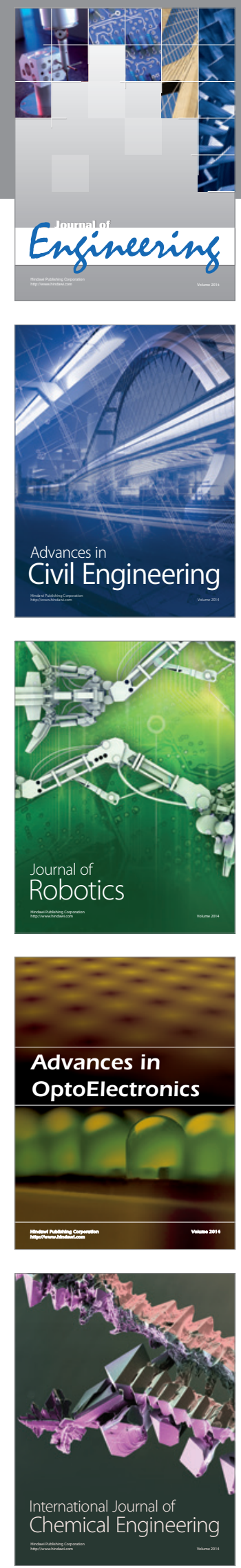

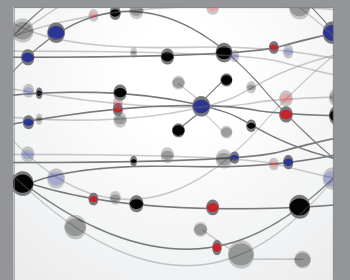

The Scientific World Journal
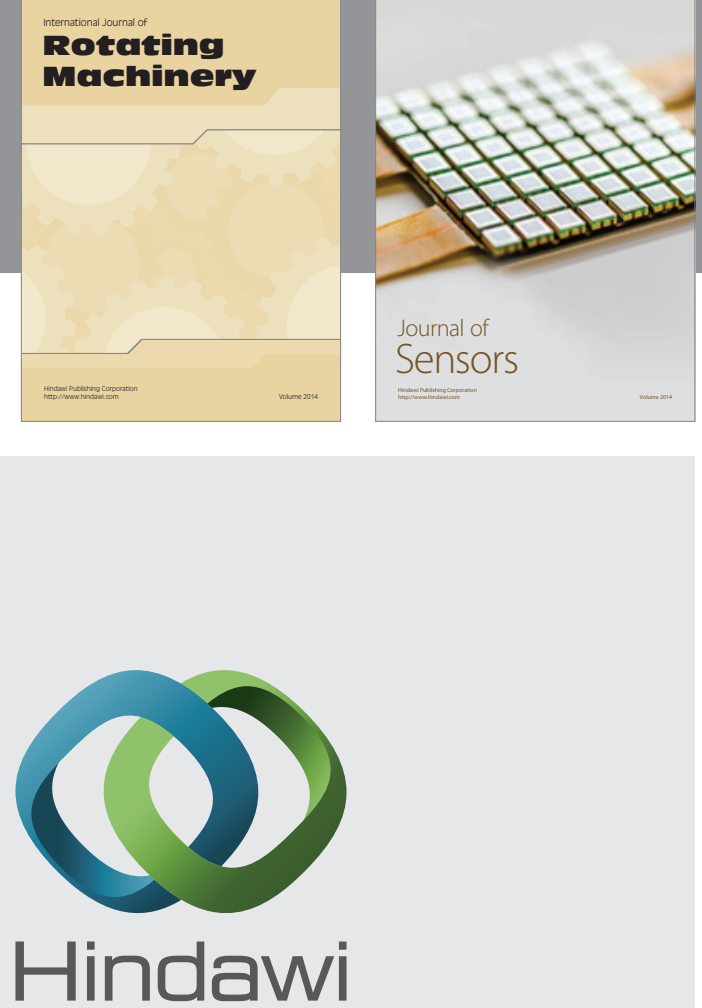

Submit your manuscripts at http://www.hindawi.com
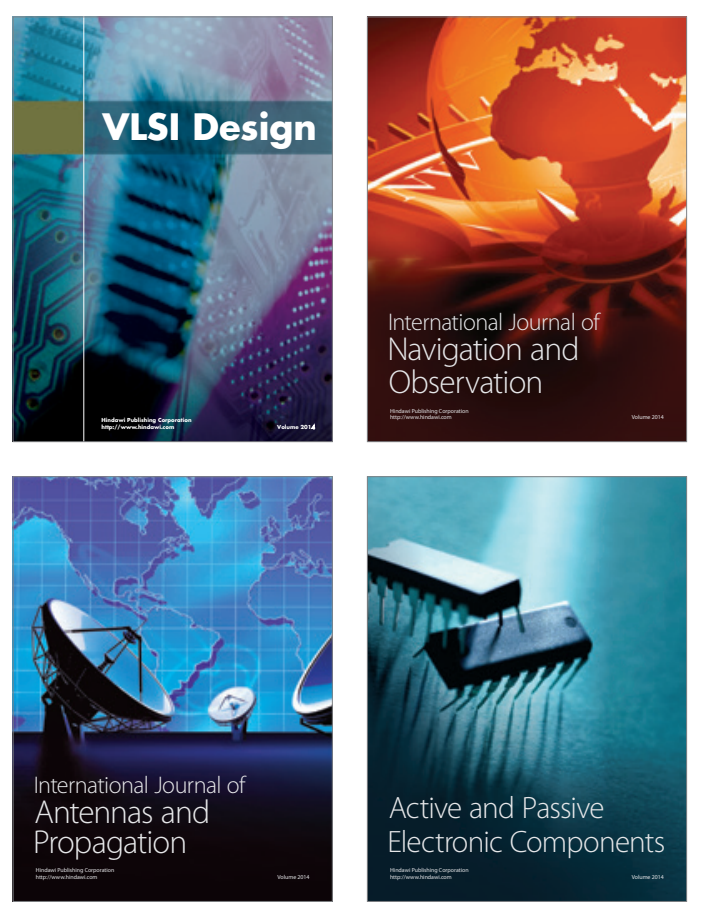
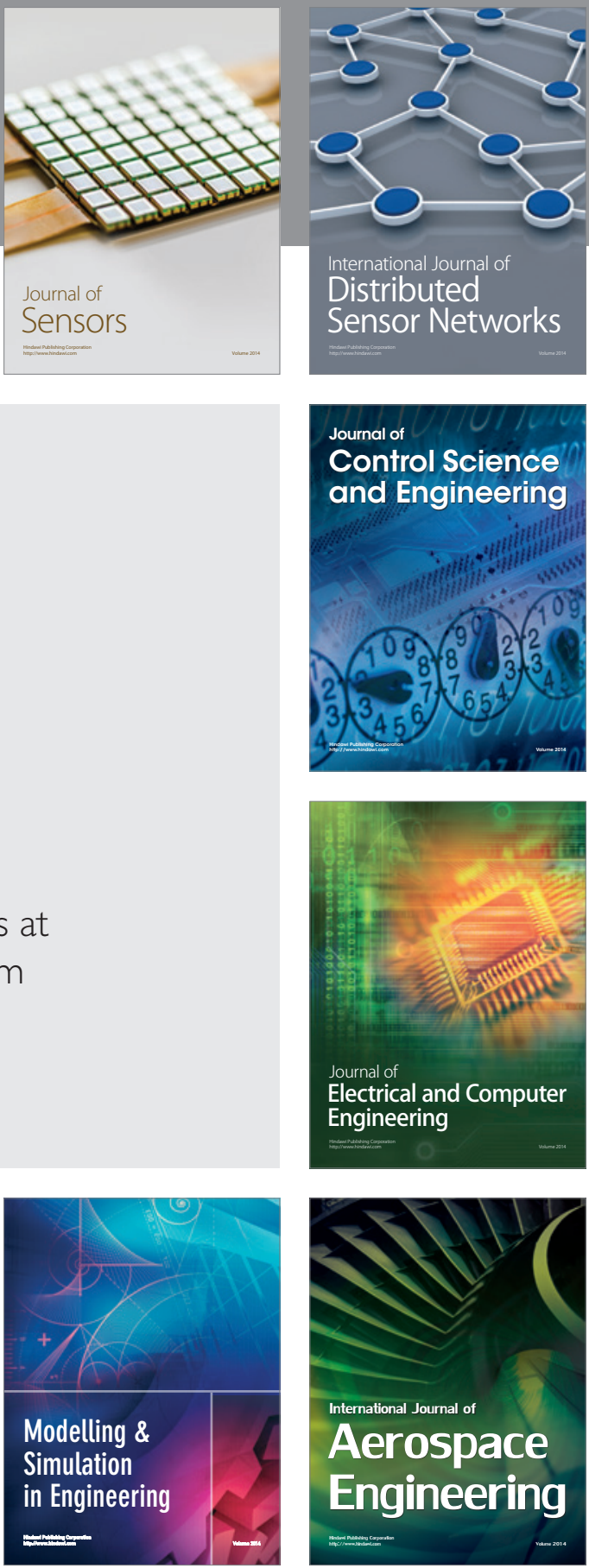

Journal of

Control Science

and Engineering
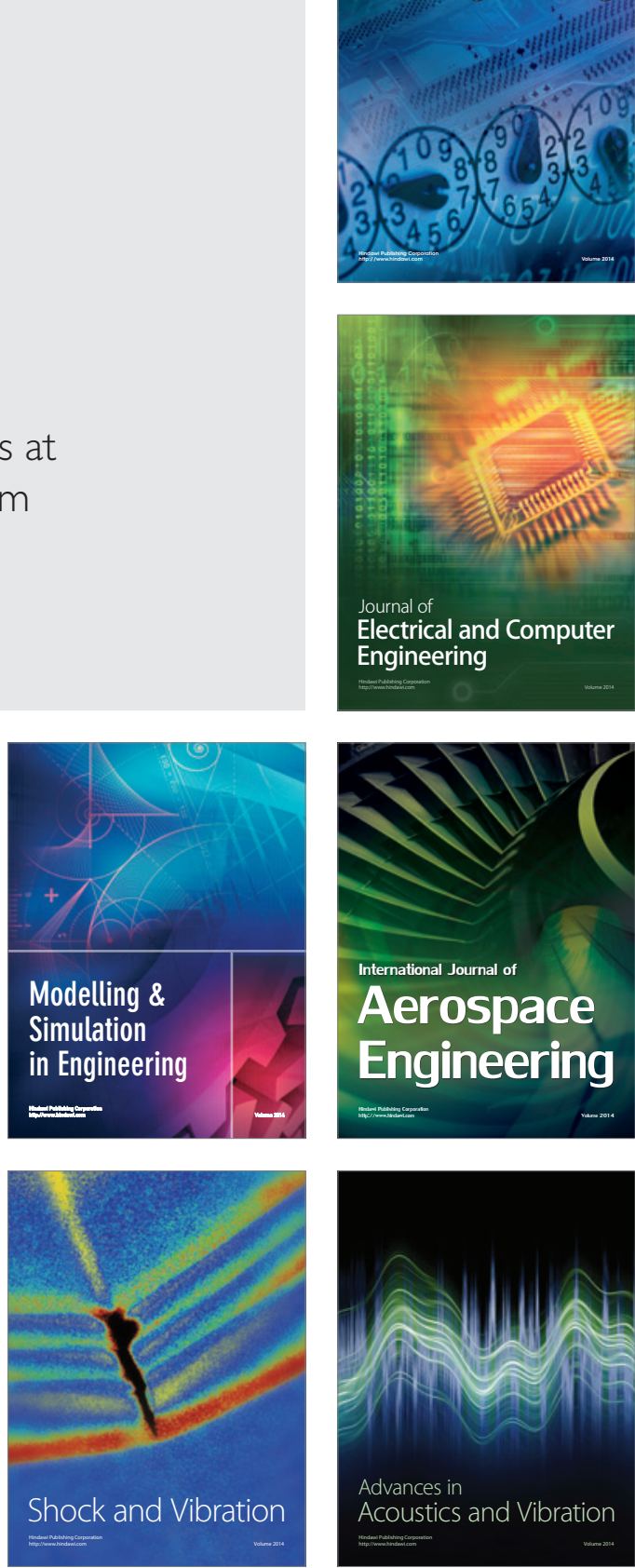